\title{
Research on the Intelligent Service System of Mass Sports under the Perspective of "Integration of Three Networks"
}

\author{
Zhiying Wang \\ Huanghe Science and Technology College \\ Zhengzhou, Henan, China 455000
}

\begin{abstract}
According to the efficiency and popularizing rate of science and technology and intelligence, this paper researches the service system of mass sports through integration of digitized information, internet function and portable mobile network. The intelligent guidance, evaluation and monitoring form effective and long lasting management, contributing to the construction of intelligent service system of mass sports. It expects the comprehensive development of sports and enlightens new research directions for the mass sports. Meanwhile, basic assumption and constructive suggestions with application and scientific research values have been proposed.
\end{abstract}

\section{Keywords—intelligence; mass sports; service system}

\section{INTRODUCTION}

In contemporary era, science and technology, informatization and internet are popular. Its development has become the inexorable trend of the mass era, inseparable from the public life. Scientific development is the only way which must be passed to promote modernization construction. With the strategic height of Guo Fa (No. 46) document, the development of "internet plus" theme and 2030 health plan requirements, the sports meet the unprecedented development opportunity. It has higher requirements and new development view for sports work. Meanwhile, it opens new development idea for the strategy of market economic system of sports cause.

Sport is only a life element for the public. It is a public welfare, sunrise and extensive industry to improve people's health. It is distinctly important to build service system, in order to better serve the public health and meet people's requirements for health. This paper builds the service system of mass sports from the perspectives of internet and intelligence. It states and analyzes how to depend on efficient, scientific, extensive and long lasting technology products to serve the public and carry out monitoring, evaluation, management and guidance of scientific physical exercise. Life and health is researched by human eternally.

The paper is sponsored by Zhengzhou Social Science Fund project. Project number (ZSLX2016597).

Project: Research on development path of public sports service system intelligence in Zhengzhou.

\section{POPUlarizing RATE AND Usage RATE OF} TECHNOLOGY PRODUCTS ESTABLISH THE FEASIBILITY RESEARCH AND DEVELOPMENT REQUIREMENTS OF INTELLIGENT SERVICE SYSTEM OF MASS SPORTS

According to the latest 2015 IC Market Driven Report issued by IC Insights, the number of mobile phone users in the world will first exceed the world's population in 2015. At that time, the world's population will exceed 7.4 billion and the number of mobile phone users will be a little more than 7.5 billion. According to China internet development statistical report, by the end of December 2014, the number of cyber citizens in our country reaches up to 649 million and the internet penetration rate is $47.9 \%$. The number of people using mobile phones for traveling reservation increases at the rate of $194.6 \%$ annually. The integrated environment and the popularizing rate of internet develop at top speed. The internet will have seven billion terminal accesses, which will certainly influence our life. Obviously, the internet becomes more indepth than extensive.

Internet and intelligence is popular among the public. The number of mobile phone users has exceeded the world's population. People depend on it because it is convenient and real-time. At present, the extensive application of internet and intelligence lays a solid foundation for the construction of intelligent service system of mass sports.

\section{THE INTEGRATION OF THREE NETWORKS IS THE INTELLIGENT DEVELOPMENT TREND OF MASS SPORTS AND THE REQUIREMENT OF TIMES}

With the soaring development of science and technology and the continuous improvement of productivity, manual labor gradually changes into brainwork. The problem that the public changes from "movement to still" becomes prominent. It violates the philosophy that life lies in movement. We physical educators should take the initiative and responsibility to solve the problem. It is the requirement of times and sports for science and technology, the requirement of the public for sports and scientific development.

Sport is life element but not necessary for the public. The mass health is the pursuit of physical educators. They need professional and systematic service system to solve problems 
in mind, will and control, looking forward to getting health standard index required by people, reasonable perceptual project, and scientific exercise prescription, frequent answer to questions as well as specific nutritional diet. The public requires a complete and reasonable service system with scientific management, monitoring, evaluation and health guidance in sports. It is the requirement of sports for the era of science and technology, the intelligent development of internet. It is our responsibility and the requirement of the public for sports development.

\section{ASSUMPTION IN THE ESTABLISHMENT OF INTELLIGENT SERVICE SYSTEM OF MASS SPORTS WITH THE INTEGRATION OF THREE NETWORKS}

\section{A. The Purpose and Importance of the Integration of Three Networks As Well As the Internet Intelligent Service System of Mass Sports}

The system is to strengthen the management of sports and the service of sport industry. It is the requirement of the public for the development of sports industry. Both enterprises and administrative departments have unique service system and specifications because it is the cultural and civilized manifestation and strengthens the influence and vitality of the industry. Meanwhile, it contributes to successful enterprises or industries in long-term development. Sport is a sunrise industry. We have more need for the establishment of intelligent service system and benign development of mass sports.

\section{B. Structure of the Integration of Three Networks and the Establishment of Intelligent Service System of Mass Sports}

This paper takes the integration of three networks as effective platform and depends on interflow, mutual assistance, mutual benefit and convenience and huge data of the internet, evaluating through data, monitoring through intelligent sports goods to form effective management pattern. We should take national authorities as the core, provincial administrative department as regional division, cities as monitoring centers to form integrated management, evaluation, monitoring and guidance of county, town, community, village and natural person.

\section{Pattern and Assumption of the Integration of Three Networks and the Establishment of Intelligent Service System of Mass Sports}

How to truly make sports popular and intelligent will be the strategic research topic. With constant improvement of needs and popularizing rate of mobile phones, it makes popular and intelligent sports become possible and develop. Taking portable mobile terminals as carriers will make it more radiant, long lasting and efficient through positioning and service of intelligent products. The portable mobile phones improve the real effect of usage rate and the integration of three networks. At present, according to its actual efficiency, corresponding medical data and functions remain to be improved. Moreover, we can take it as monitoring instrument to remind, warn and give information required for the public, so that they can know about their health. Data required are given to them scientifically and reasonably. The community sports instructors can provide door-to-door service with village and community as basic unit, town as supervision station, province and cities as management center. We can depend on professional research institutes in university to give theoretical guidance through establishing database, personal health record and giving health data. Scientific health guidance and exercise prescription are provided for demanders, in order to effectively enhance the development of mass sports and health management. At the same time, we can exploit products in the market of mass sports according to the data in health management, thus drive the industrialization development of sports market and meet the requirements of people for mass sports.

V. THE Establishment OF INTELLIGENT SERVICE SYSTEM OF MASS SPORTS VIA THE INTEGRATION OF THREE NETWORKS AND VALUE ADVANTAGE

\section{A. The Intelligent Service System of Mass Sports with the Integration of Three Networks Has Improved the Efficient Management of Mass Sports.}

It is well known that China has vast territory and abundant resources as well as enormous population. It is very difficult to realize centralized management. People have a wide distribution and the distance is one of the adverse factors. We must establish an efficient platform to cover extensive media and interconnect factors in the space. Internet is the best platform which breaks through the obstacle, creates space value and improves effectiveness, intuition and resource sharing. It will be conducive to the development of mass sports, centralized management, overall arrangement, so that we can realize scientific and systematic management and efficient value.

\section{B. The Intelligent Service System of Mass Sports with the Integration of Three Networks Strengthens the Penetration of Mass Sports Culture}

Contemporary sports focus on athletics. It is difficult to achieve unified management and arrangement and service of mass sports because of the enormous population, differences in ethnic and traditional culture, weakened sport consciousness and geographic region. These problems influence our right to take physical exercise even marketization and industrialization development of sports. The establishment of internet and intelligent service system of mass sports will roundly and effectively promote the sports development, because everyone can access to it. A mobile phone or intelligent product can monitor and evaluate the indicators of human body. According to the monitoring and evaluation, we provide corresponding countermeasures and give some guidance. The system will certainly improve and meet the needs of the public for different sports. Therefore, the popularization of service system makes for the all-round development of the whole sports field. 


\section{The Integration of Three Networks Makes for the Establishment of Sound Intelligent Service System of Mass Sports}

A sound management system should be extensive. With the development of sports industry, the service of sports has become a new trend. It has become the core goal and strategic emphasis of sports. Therefore, it is of vital importance to establish service system of sports, especially in the transformation period. It becomes the primary problem about how to systematically establish development pattern. The establishment of internet and intelligent service system can guarantee effective work, save manpower resource and ease the economic pressure of the government. Furthermore, it drives the development of sports scientific research and industry and activates the sports industry in management, evaluation and monitoring of training, guidance and competition required in sports. The research on intelligent service system of mass sports can improve the industrialization structure of sports and balance the technology and the theory of sports.

\section{CONCLUSION}

Health is the substantial need of the public. The scientific research of sports should pay the most attention to it. The mass health with the assistance of sports directly reflects the industry and state politics, especially in remote and border areas with backward sports. The concept of internet plus sports will be the concentrated reflection of deepening reform of sports. This paper assumes and establishes research ideas of service system according to internet intelligent products, proposing new methods in intelligent monitoring, evaluation and management of mass sports with the help of internet. It is an important problem in urgent need of reform and in-depth study for sports and mass health. .

\section{REFERENCES}

[1] Li Fei. Research on the Mobile Service Mode of Digital Library under the Perspective of Integration of Three Networks [A], Doctoral Thesis, May 2015

[2] Cui Qian. Research on the Construction of Service System with Diversified and Intelligent Development for Community Sports of Baoji City [J], Forum on Science and Technology in China, 2013(23): 287288

[3] Liu Meijin. The Internet Will Certainly Promote the Sports Science and Technology to Move towards Healthy Families [D], Beijing Sport University, Dec.2013

[4] Shu Yiming, Zhang Di. Research on the Informatization and Intelligent Process of the Scientific Development of Sports [J], Journal of Chifeng University, Jul.2013: 145-146

[5] Wang Zhuli, Li Xiaoyu, Lin Jin. Smart Phone and Internet plus Classroom [J], Distance Education Journal, Apr.2015: 14-21

[6] Si Hongbo, Shen Yan, Ma Wenfeng. Analysis on the Value of People's Livelihood of Sports Development [J], Theory and Practice of Contemporary Education, Jul.2015: 161-163

[7] Xiang Huiying. Development of Social Organizations of Sports under the Background of Deepening Reform in the 13th Five-year Plan [J], Sport Science Research, Mar.2015: 36-44

[8] Zhang Mingjun. Crisis of Traditional Management in Our Country and Governance and Construction of the New Type Sports [J], Journal of Physical Education, Jul.2015: 13-16
[9] Wei Qiang. Analysis on Bottleneck in Transformation of Sports Power [J], Sports World, Jul.2015:10-11+19

[10] Feng Xiaoli. Research Progress and Development Path of Folk Sports Organizations in Our Country [J], Sports Research and Value, Aug.2015: $1-8$. 